\title{
Double-Network-Structured Graphene Oxide-Containing Nanogels as Photothermal Agents for the Treatment of Colorectal Cancer
}

\author{
Calogero Fiorica, ${ }^{\dagger}$ Nicolò Mauro, ${ }^{*}{ }^{\dagger}$ Giovanna Pitarresi, ${ }^{\dagger}$ Cinzia Scialabba, ${ }^{\dagger}$ Fabio S. Palumbo, ${ }^{\dagger}$ \\ and Gaetano Giammona ${ }^{\dagger, \ddagger}$
}
${ }^{\dagger}$ Laboratory of Biocompatible Polymers, Department of "Scienze e Tecnologie Biologiche, Chimiche e Farmaceutiche" (STEBICEF), University of Palermo, Via Archirafi, 3290123 Palermo, Italy
${ }^{\ddagger}$ Mediterranean Center for Human Advanced Biotechnologies (Med-Chab), Viale delle Scienze Ed.18, 90128 Palermo, Italy

\section{Supporting Information}

ABSTRACT: Here, we reported the production of hyaluronic acid/polyaspartamide-based double-network nanogels for the potential treatment of colorectal carcinoma. Graphene oxide, thanks to the huge aromatic surface area, allows to easily load high amount of irinotecan $(33.0 \% \mathrm{w} / \mathrm{w})$ and confers to the system hyperthermic properties when irradiated with a nearinfrared (NIR) laser beam. We demonstrate that the release of antitumor drug is influenced both by the $\mathrm{pH}$ of the external medium and the NIR irradiation process. In vitro biological studies, conducted on human colon cancer cells (HCT 116),

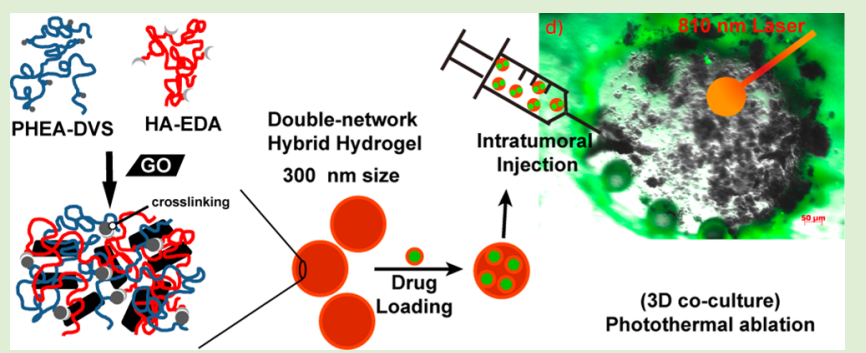
revealed that nanogels are uptaken by the cancer cells and, in the presence of the antitumor drug, can produce a synergistic hyperthermic/cytotoxic effect. Finally, 3D experiments demonstrate that it is possible to conduct thermal ablation of solid tumors after the intratumoral administration of nanogels.

\section{INTRODUCTION}

Colorectal cancer (CRC) is one of the most common cancers in developed countries, with an incidence in Europe of 471000 new cases and 228000 deaths in the year 2012. ${ }^{1,2}$ Stages 0 and I refer to early phase tumors for which, in the majority of cases, surgical resection is the only treatment needed, ${ }^{3}$ while an adjuvant chemotherapy is performed after surgery for those patients with stage II and stage III CRC. Currently, the most employed antitumor drugs for the treatment of CRC are 5fluoruracil, capecitabine, irinotecan (IT), and oxaliplatin. The systemic administration of these cytotoxic agents is associated with several drawbacks mainly attributable to the distribution in the healthy tissues and their poor pharmacokinetic profiles. Moreover, it has been recently reported that a high percentage (about 50\%) of patients with CRC develop recurrent disease despite surgical resection and adjuvant chemotherapy treatment. ${ }^{4}$

In the last decades, nanomedicines have been considered as a promising alternative to the classic antitumor drug dosage forms thanks to their ability to modify the biodistribution, tissue uptake, and pharmacokinetics of therapeutic agents. ${ }^{5}$ Nanosized systems can be directed selectively to the tumor through the "enhanced permeability and retention" effect and through the functionalization with active targeting ligands. Nanomedicines can release their drug payload nearby the site of action and increase its effectiveness. ${ }^{\text {- }}$

Active targeting can also be obtained by producing the nanosystems employing as starting biomaterials molecules that can be recognized by cancer cells. For example, hyaluronic acid
(HA), a natural glycosaminoglycan widely distributed in the human tissues, has been employed to produce nanoparticles and micelles as colloidal carriers for target-specific delivery of anticancer drugs thanks to its peculiar property to be recognized by cellular receptors as CD44 and RHAMM glycoproteins that are overexpressed by the cells of several tumors ${ }^{1-14}$ as breast, ovarian, and colon cancer.

Recently, hybrid nanosystems like iron/gold nanoparticles and graphene derivatives have been proposed as selective therapeutic tools for the treatment of several kinds of tumors and also for CRC. ${ }^{15-17}$ Graphene derivatives have aroused a particular interest because of their remarkable properties as a drug delivery systems and also, as recently discovered, as hyperthermia agent for anticancer therapies. ${ }^{18}$ This last aspect is particularly interesting since hyperthermia, intended as a local rise in temperature into the tumor mass (usually to $40-45^{\circ} \mathrm{C}$ ), in addition to directly causing the death of tumor cells, is able to potentiate the cytotoxic effect of many anticancer drugs, ${ }^{19}$ among which IT, as demonstrated by Cotte at al. ${ }^{20}$

To date, hyperthermia is considered an almost essential adjuvant practice in chemotherapy, since it seems that the administration of nanomedicines that act with only one therapeutic mechanism may lead to drug resistance phenomena, especially for the treatment of disseminated tumor masses. ${ }^{21}$ In this scenario, by focusing the attention on the

Received: December 21, 2016

Revised: February 3, 2017

Published: February 13, 2017 
CRC treatment, it is clear that there is still a strong demand to develop new smart multitask nanostructures capable of performing the therapeutic effect by combining a selective cytotoxic effect, the capability to carry out a synergistic therapeutic effect, and eventually, facilitate the detection of the tumor cells. With this in mind, here, we describe the production of HA-based nanogels $(\mathrm{NG})$ containing graphene oxide (GO) to be exploited for the incorporation and the delivery of IT for the treatment of CRC.

We demonstrated that the presence of GO allows to incorporate a high amount of IT and gives to the NG hyperthermic properties when irradiated with a cold laser beam.

Erythrolysis studies showed that obtained NG was safe when in contact with the cellular component of blood, highlighting their potential intravenous administration. Through $2 \mathrm{D}$ in vitro experiments, it was observed that the hyperthermic effect increases drastically the cytotoxic effect of the antitumor drug toward human colon cancer cells (HCT 116), while 3D experiments, conducted with a coculture of cancer and healthy cells, demonstrate that it could be possible to directly ablate the tumor mass after local injection of the NG.

\section{MATERIALS AND METHODS}

Materials. Sodium hydroxide (99.5\%), aspartic acid (98\%), ethylenediamine (99\%), bis(4-nitrophenyl) carbonate (99\%), and irinotecan hydrochloride (IT; 99\%) where purchased from SigmaAldrich and used as received. Single layer dispersion graphene oxide was purchased from ACS Material (U.S.A.) and used after purification by dialysis tube with nominal molecular weight cutoff $100 \mathrm{kDa}$ : flake size $500 \mathrm{~nm}$, thickness $0.8-1.1 \mathrm{~nm}$, oxidation degree $>45 \%$. SpectraPor dialysis tubing was purchased from Spectrum Laboratories, Inc. (Italy).

Cell Tracker Green (5-chloromethylfluorescein diacetate dye) was purchased by Thermo Fisher Scientific.

Human venous plasma and uncoagulated blood were obtained from healthy voluntary donors, in compliance with the Italian laws and institutional guidelines, and immediately used. In particular, these samples were collected from the first coauthor with his informed consent and the approval of STEBICEF scientific committee.

Preparation of the HA-EDA-PHEA-DVS/GO Composite Nanogel (NG). Hyaluronic-((2-aminoethyl)-carbamate) acid (HAEDA) and $\alpha, \beta$-poly $(N$-2-hydroxyethyl)-D,L-aspartamide-divinyl sulfone (PHEA-DVS) were synthesized as reported elsewhere. ${ }^{22,23}$ A hydrogel of HA-EDA $(150 \mathrm{mg}$ ) was obtained dissolving the polymer in water $(2.5 \mathrm{~mL}$ ) by sonicating for $5 \mathrm{~min}$ followed by a lively stirring for $5 \mathrm{~min}$ $(6 \times)$. The complete dissolution was obtained keeping this dispersion at $40{ }^{\circ} \mathrm{C}$ for $12 \mathrm{~h}$. After that, separately, $2 \mathrm{~mL}$ of a solution of GO $(5$ $\left.\mathrm{mg} \mathrm{mL}{ }^{-1}\right)$ were added to PHEA-DVS $(150 \mathrm{mg})$ in water $(1 \mathrm{~mL})$. This solution was then added to the HA-EDA hydrogel and the $\mathrm{pH}$ was adjusted to 10.0 using $2 \mathrm{M} \mathrm{NaOH}(20 \mu \mathrm{L})$. A total of $18 \mathrm{~h}$ after incubation at $25{ }^{\circ} \mathrm{C}$ the crude hydrogel was roughly homogenized by means of a Ultra-Turrax ( $5 \mathrm{~min}, 17.500 \mathrm{rpm}, 20^{\circ} \mathrm{C}$, 4 times), washed with water four times, and then homogenized by using a high pressure homogenizer (HPH; Microfluidizer M-110P, Microfluidics): $30 \mathrm{kpsi}, 5$ cycles, $20^{\circ} \mathrm{C}$. Finally, NG was retrieved as a transparent pellet through ultracentrifugation: $40000 \mathrm{rpm}, 1 \mathrm{~h}, 10^{\circ} \mathrm{C}$.

Preparation of the CY5-Labeled NG. The composite NG was obtained as above-described, but using a CY5-hydrazide labeled (Alexafluor; $\lambda_{\mathrm{Ex}}{ }^{646}, \lambda_{\mathrm{Em}}{ }^{664}$ ) GO, obtained incubating a solution of GO $\mathrm{pH} 6.8\left(5 \mathrm{mg} \mathrm{mL}^{-1}\right)$ with the fluorescent probe $(0.1 \mathrm{mg})$ and a mixture of EDC/NHS $(1: 1 \mathrm{mg})$ for $6 \mathrm{~h}$. After this time CY5-labeled $\mathrm{GO}$ was purified by dialysis to remove the free marker.

Dynamic Light Scattering and Z-Potential of NG. Average size, size distribution, or polydispersity index (PDI) and Z-potential measurements were determined by dynamic light scattering (DLS) at $25{ }^{\circ} \mathrm{C}$ using a Malvern Zetasizer NanoZS instrument fitted with a $532 \mathrm{~nm}$ laser at a fixed scattering angle of $173^{\circ}$. Aqueous solutions of
NG $\left(0.1 \mathrm{mg} \mathrm{mL}^{-1}\right)$ were analyzed after filtration through a $5 \mu \mathrm{m}$ cellulose membrane syringe filter. The $Z$-potential $(\mathrm{mV})$ was calculated from the electrophoretic mobility using the Smoluchowsky relationship and assuming that $K a \gg 1$ (where $K$ and $a$ are the Debye-Huckel parameter and particle radius, respectively). Data are the mean $\pm \mathrm{SD}$ of $n=6$ independent experiments.

Morphological Characterization of the NG. The morphology studies of the NG scanning electron (SEM) and atomic force microscopies (AFM) were employed. For the SEM analysis, a drop of a NG dispersion in water $\left(0.1 \mathrm{mg} \mathrm{mL}^{-1}\right)$ was deposed onto a carbon adhesive previously applied on a stainless steel stub. It was then dried under vacuum (40 mbar) for $24 \mathrm{~h}$. Hence, the sample was sputtercoated with gold prior to microscopy examination. Micrographs were recorded using a scanning electron microscope, ESEM Philips XL30.

AFM images were obtained in Tapping mode in air by a Bruker Dimension FastScan microscope equipped with closed-loop scanners. We used triangular FastScan A probes (resonance frequency $=1400$ $\mathrm{kHz}$, tip radius $=5 \mathrm{~nm})$. An aqueous dispersion of $\mathrm{NG}(0.001 \mathrm{mg}$ $\mathrm{mL}^{-1}$ ) was dropped onto a freshly cleaved mica surface and dried overnight before observation.

Swelling Degree Kinetic. Swelling experiments were performed in water on dried NG sample $(\approx 10 \mathrm{mg})$. Dried samples where obtained putting the swollen NG in a vacuum chamber (0.1 Torr) until constant weight. Each sample was put into a test tube containing $40 \mathrm{~mL}$ of water and maintained at $25{ }^{\circ} \mathrm{C}$ for $12 \mathrm{~h}$. At regular time intervals the sample was retrieved by centrifuging, weighed, and reintroduced into the test tube. Maximum swelling was observed after about $2 \mathrm{~h}$. The percent mean variation coefficient, CV\%, was lower than $5 \%$. The reported values were the average of six experiments.

Drug Loading and Drug Release. The incorporation of IT hydrochloride $(20 \mathrm{mg})$ was carried out in water $(5 \mathrm{~mL})$ by sonicating and stirring the swollen NG $(760 \mathrm{mg})$ for 15 and 30 min respectively (5 cycles). NG was purified by the free drug through ultracentrifugation: $1 \mathrm{~h}, 40000 \mathrm{rpm}, 10{ }^{\circ} \mathrm{C}$. The amount of IT loaded into the IT/NG was evaluated spectrophotometrically (Shimadzu UV Spectrophotometer) using a calibration curve obtained by measuring the absorbance of reference standards in DMSO at $\lambda 383 \mathrm{~nm}\left(R^{2}=\right.$ 0.997). The extraction of the drug from the IT/NG $(5 \mathrm{mg})$ was performed in DMSO $(5 \mathrm{~mL})$ by means of sonication for $10 \mathrm{~min}$, followed by stirring for $3 \mathrm{~h}$. The sample was filtered through a syringe filter $(0.45 \mu \mathrm{m})$ before performing the UV measures. The drug loading (DL\%) was 33\% with an encapsulation efficiency of $100 \%$ (amount of drug loaded/theoretical drug loading percentage ratio, on a weight basis).

For drug release studies the IT/NG $(3.0 \mathrm{mg})$ was dissolved in either $\mathrm{PBS}$ at $\mathrm{pH} 7.4$ or $\mathrm{pH} 5.5(5 \mathrm{~mL})$ and placed into a dialysis tubing with a MWCO $12-14 \mathrm{kDa}$. It was then immersed into the medium (40 $\mathrm{mL})$ and incubated at $37^{\circ} \mathrm{C}$ under continuous stirring $(100 \mathrm{rpm})$ in a Benchtop 808C Incubator Orbital Shaker model 420, for $12 \mathrm{~h}$. Aliquots of the external medium $(1 \mathrm{~mL})$ were withdrawn from the outside of the dialysis tubing at scheduled time intervals and replaced with equal amount of fresh medium. The amount of IT released was evaluated by HPCL (C6-phenyl column; $0.15 \mathrm{mM}$ phosphate buffer

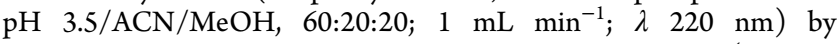
comparison with a standard curve of IT hydrochloride (linearity range $\left.=10^{-3}-0.5 \mathrm{mg} \mathrm{mL}^{-1} ; R^{2}=0.988\right)$. Then, the cumulative release was determined as a function of incubation time.

Evaluation of the Hyperthermic Effect of NG. A dispersion of NG in water $\left(20 \mathrm{~mL}, 0.25 \mathrm{mg} \mathrm{mL}^{-1}\right)$, was prepared and treated with a $810 \mathrm{~nm}$ diode laser (GBox 15A/B by GIGA Laser) with the power fitted at $3 \times 10^{-3} \mathrm{~W} \mathrm{~mm}^{-3}$. At fixed intervals the temperature of the dispersion was recorded and reported as a function of the exposure time.

Uptake, Cytotoxicity, and Erythrolysis Studies. Uptake studies were conducted as follow: HCT-116 $\left(1 \times 10^{4}\right)$ were plated in 98-well plate and cultured for $24 \mathrm{~h}$, at $37{ }^{\circ} \mathrm{C}$ and $5 \% \mathrm{CO}_{2}$ atmosphere, with DMEM supplemented with fetal bovine serum $(10 \% \mathrm{v} / \mathrm{v})$, glutamin, penicillin-streptomicin solution, and amphotericin. After $24 \mathrm{~h}$, the medium was changed with $\mathrm{Cy}$-5-labeled NG suspension $(0.25 \mathrm{mg}$ $\mathrm{mL}^{-1}$ ) and the samples were incubated for further 4 and $24 \mathrm{~h}$. After 
Scheme 1. Schematic Representation of the Processes Involved in the Preparation of NG

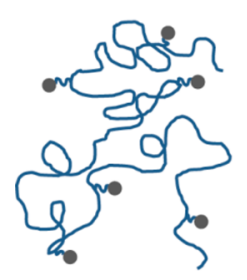

PHEA-DVS

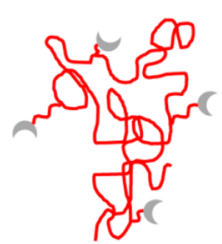

HA-EDA
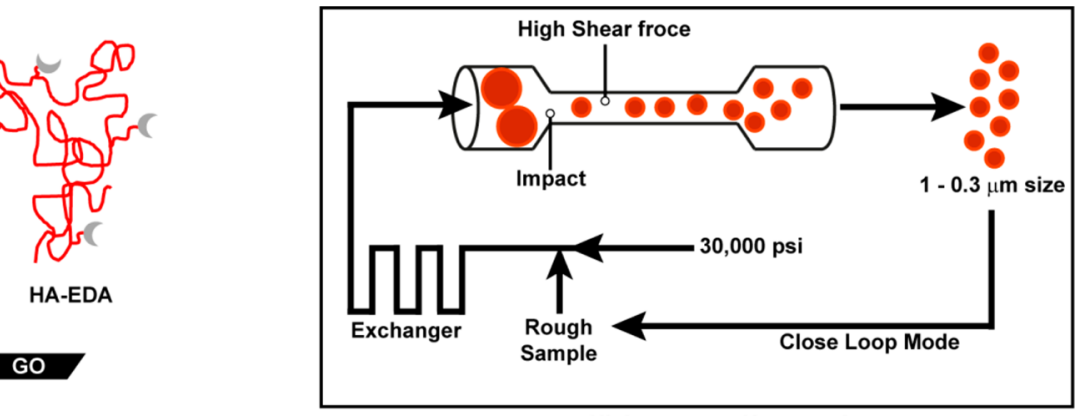

High-pressure Homogenize
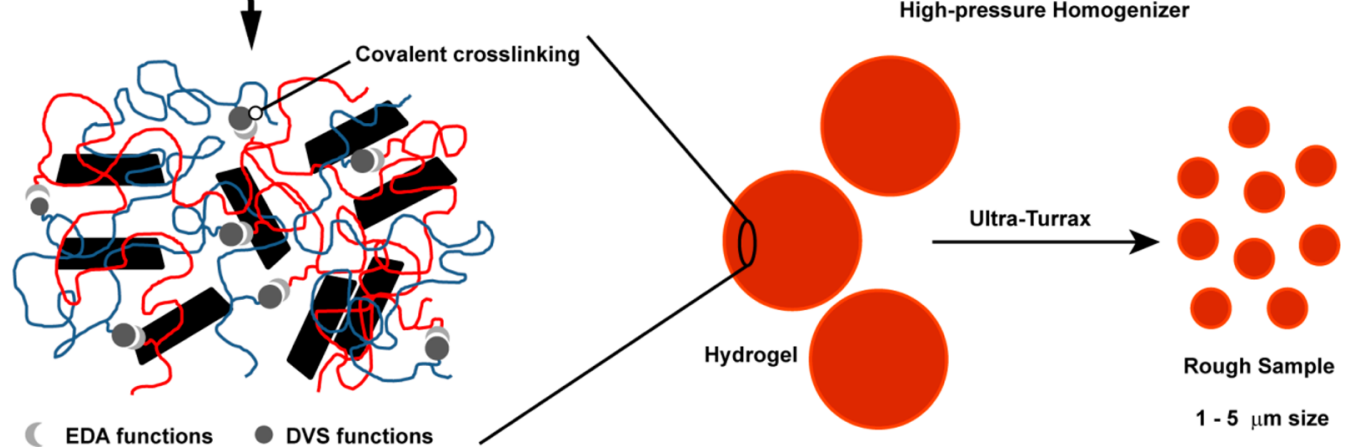

scheduled times the NG suspensions were withdrawn and cells were washed three times with PBS $\mathrm{pH}$ 7.4. Fluorescence microscopy measurements were performed by using a Zeiss microscope (AXIO Vert.A1) and by using a $20 \times$ air objective and proper filters for excitation $(625-655 \mathrm{~nm})$ and emission lights $(660-715 \mathrm{~nm})$. A flow cytometry analysis was also performed to give a quantitative insight of the uptake process according with a protocol reported by Allegra et al. ${ }^{24}$ Briefly, for this analysis, $4 \times 10^{4}$ were plated in 24 well plate and cultured for $24 \mathrm{~h}$ prior to change the medium with $\mathrm{Cy}$-5-labeled $\mathrm{NG}$ suspension $\left(0.25 \mathrm{mg} \mathrm{mL}^{-1}\right)$. At the scheduled times ( 4 and $24 \mathrm{~h}$ ), cells were collected by centrifugation for $7 \mathrm{~min}$ at $1000 \mathrm{rpm}$, washed, suspended in PBS and immediately analyzed with an EPICS XL cytofluorimeter (Beckman Coulter Inc., U.S.A.) with FL4 channel. At least $1 \times 10^{4}$ cells were analyzed for each sample.

Cytotoxicity studies were conducted by employing DMEM suspensions of $\mathrm{NG}$ or IT/NG, with IT concentration ranging from 25 to $400 \mu \mathrm{g} \mathrm{mL}^{-1}$, as culture medium for human colorectal cancer cells (HCT 116). Cells were seeded with the same conditions already described for the uptake experiments.

After $24 \mathrm{~h}$, the medium was changed with the NG or IT/NG suspensions. HCT 116 cultured in supplemented DMEM were used as positive control while DMEM solutions of IT with the same concentrations of those in the IT/NG were employed as negative control. The viability of the cells was monitored after 4 and $24 \mathrm{~h}$ of incubation through MTS (3-(4,5-dimethylthiazol-2-yl)-5-(3-carboxymethoxyphenyl)-2-(4sulfophenyl)-2H-tetrazolium) assay (CellTiter 96 AQueous One Solution Cell Proliferation, PROMEGA) following the manufacturer instructions.

For the erythrolysis studies, erythrocytes were collected by centrifuging $2 \mathrm{~mL}$ of fresh blood at $2200 \mathrm{rpm}$ for $10 \mathrm{~min}$ and, then, by washing up the red pellet with PBS at $\mathrm{pH} 7.4$ several times until a colorless aqueous supernatant was observed. A 4\% v/v suspension of erythrocytes in PBS $\mathrm{pH} 7.4(200 \mathrm{~mL})$ was added to a $\mathrm{NG}$ suspension at concentration of $1 \mathrm{mg} \mathrm{mL}^{-1}$. The suspension was incubated at $37 \mathrm{C}$ for $1 \mathrm{~h}$, centrifuged at $2200 \mathrm{rpm}$ for $5 \mathrm{~min}$ and the amount of hemoglobin released in the supernatant was measured spectrophotometrically at $570 \mathrm{~nm}$. Triton-X $100 \mathrm{DMEM}$ solution (1\% w/v) (Sigam Aldrich) was used as a positive control in order to normalize all data.

Hyperthermic Effect onto HCT 116. DMEM suspensions of NG with different concentrations (ranging from 0.0625 to $1 \mathrm{mg} \mathrm{mL}^{-1}$ ) were incubated with HCT 116 seeded in 96-well plates $24 \mathrm{~h}$ earlier (1 $\times 10^{4}$ cells per well). The plates were incubated at $37{ }^{\circ} \mathrm{C}$ and $5 \% \mathrm{CO}_{2}$ atmosphere and, after 4 and $24 \mathrm{~h}$, each well was irradiated with laser (employing the same settings already described for the evaluation of the hyperthermic effect of NG aqueous suspensions) for 100 or $200 \mathrm{~s}$. After the laser treatment, the viability of the cells was evaluated with MTS and expressed as percentage of the viability of HCT 116 cultured in DMEM that did not undergo the laser treatment. Hyperthermia experiments were conducted also by irradiating samples in contact with IT/NG suspensions. For these experiments, 0.0625 and $0.125 \mathrm{mg}$ $\mathrm{mL}^{-1}$ IT/NG suspensions were kept in contact with HCT 116 (seeded as described for the previous experiments) for $4 \mathrm{~h}$. After that, the samples were irradiated for 100 or $200 \mathrm{~s}$ prior to evaluating the viability of the cells with MTS. Samples treated with free IT solutions at concentrations of 25 and $50 \mu \mathrm{g} \mathrm{mL}^{-1}$ were also irradiated and employed as negative control. The viability of all the samples was expressed as percentage of that of HCT 116 cultured in DMEM that did not undergo the laser treatment.

3D Tumor Model and Thermal Ablation Assay. The 3D solid tumor model was developed by culturing both human dermal fibroblasts (HDF) and HCT 116 in Matrigel. The constructs were prepared as follows: $1 \times 10^{6} \mathrm{HDF}$ were pelleted and resuspended in $500 \mu \mathrm{L}$ of fluid Matrigel at a temperature of $18{ }^{\circ} \mathrm{C}$ and the obtained cell suspensions were left at room temperature until the formation of hydrogels occurs. A total of $50 \mu \mathrm{L}$ of complete DMEM containing $5 \times$ $10^{5}$ Cell Tracker Green stained HCT 116 (stained following the manufacturer specifications) were then inoculated with a syringe into each hydrogel. The 3D constructs were cultured in complete DMEM for $24 \mathrm{~h}$ prior to perform further assays. For the thermal ablation assay, $200 \mu \mathrm{L}$ of $0.125 \mathrm{mg} \mathrm{mL}^{-1}$ of NG or IT/NG were inoculated into the 3D construct with a syringe. Samples were incubated for $4 \mathrm{~h}$ and then subjected to laser irradiation for $200 \mathrm{~s}$ directing the laser beam into the mass of tumor cells. The evaluation of the irradiation effect was performed by fluorescence microscopy $24 \mathrm{~h}$ after the laser treatment.

\section{RESULTS AND DISCUSSION}

Preparation of the HA-EDA-PHEA-DVS Nanogel (NG). Nanogels are described as hydrophilic three-dimensional polymer networks confined to nanoscopic dimensions that are able to take up large amounts of aqueous media, while maintaining their network structure. ${ }^{25}$ In the past decade there has been increasing attention in nanogels, since they can be designed to facilitate the encapsulation of a huge class of 
bioactive compounds to be safely delivered into the cytoplasm of cells. ${ }^{26-28}$ However, having high water uptake and fast network medium turnover, only hydrophilic actives can be encapsulated in nanogels and usually they diffuse rapidly throughout, thus limiting the use of this nanomedicine in targeted therapy. ${ }^{29}$ In this study, we demonstrate the introduction of GO nanosheets in the nanogel network allows high loading capability of poorly soluble anticancer drugs (i.e., IT), thereby increasing their chances to be accumulated in the tumor site than the free drug. Unlike the existing strategies for the preparation of nanogels, which commonly exploit the physical self-assembly of polymers possibly stabilized by means of chemical cross-linking of preformed polymers, ${ }^{30}$ we employed a two-step highly scalable top down method, which permits the fine control of particle size, composition, and dispersity. Here, we built up a hydrophilic barrier around single GO sheets consisting of hyaluronic acid, thus acting as lyophilic colloid so as to stabilize GO dispersion in aqueous media and to serve CD44+ targeting agent toward colon rectal carcinoma. $^{31}$

In particular, a bulk nanocomposite hydrogel was first obtained by Michael-type addition of an amino-functionalized HA $\left(M_{\mathrm{w}}=100 \mathrm{k}\right.$; HA-EDA $)$ with $\alpha, \beta$-poly(N-2-hydroxyethyl)D,L-aspartamide-divinyl sulfone $\left(M_{\mathrm{w}}=38 \mathrm{k}\right.$; PHEA-DVS $)$ in the presence of single layer GO at $\mathrm{pH} 10.0$ (Scheme 1). The amine functions of HA-EDA were exactly balanced by DVS functions of PHEA-DVS, thus, providing a rigid network without excess both double bond and amines. As previously described, this dual polymeric network brings about higher mechanical stiffness though keeping the high water uptake typical of hyaluronane, making it more applicable for nanomedicine applications. ${ }^{23}$ In order to produce nanogels (NG), the bulk hydrogel was, in turn, homogenized by ultraturrax and high pressure homogenizer $(\mathrm{HPH} ; 30 \mathrm{kpsi})$, obtaining a stable particle dispersion after purification with water. Dynamic light scattering measurements revealed that a strong reduction of the NG particle size occurred after the HPH treatment (Z-average passes from 1900 to $300 \mathrm{~nm}$ ), accompanied by a sharp decreasing of the polydispersity index (from 0.75 to 0.22 ), showing also that no significant changes can be registered after three cycles of homogenization (Figure la and Table 1). ZPotential value increased as a function of the NG dimension $(-17$ vs $-24 \mathrm{mV})$ with an outstanding beneficial effect on the NG stability in physiological media (Table 1).

Shape is a useful parameter for effective drug delivery particle design (probably more important for successful cell engulfment than target size). For instance, biodistribution studies have demonstrated that particles with high aspect ratios successfully evade phagocytosis and are endowed with prolonged blood circulation if compared with spherical particles of similar diameter. $^{32,33}$ Hence, polygonal nanoparticles, as a rule, outperform nanospheres in terms of extravasation into tumors, since nanoparticle extravasation into tumors is proportional to their bioavailability. ${ }^{34}$ Taking these in mind, the morphology of the NG particles was studied by coupling scanning electron microscopy (SEM) and atomic force microscopy (AFM; Figure $1 \mathrm{~b}$ and $\mathrm{c}$, respectively). Both techniques showed polygonal nanoparticles with dimension below $400 \pm 40 \mathrm{~nm}$ in diameter, thus, confirming DLS data obtained in the swollen state.

Moreover, the direct observation of the sample did not reveal any free GO flake, assuming that GO was completely incorporated inside the NG network. On the contrary, AFM micrographs highlighted the presence of GO sheets with a a

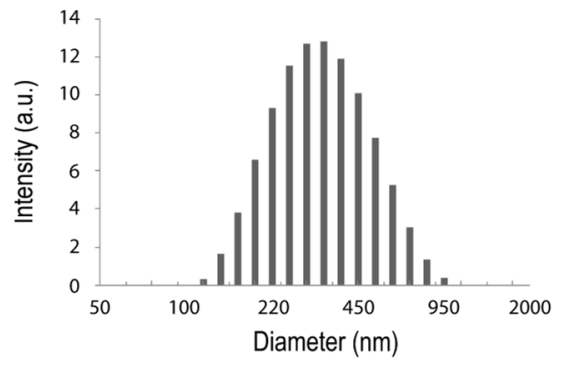

b

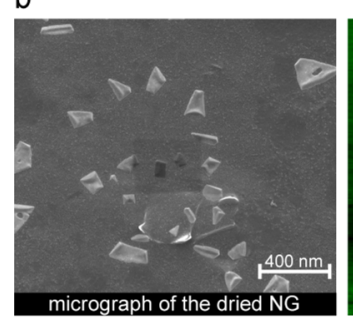

C

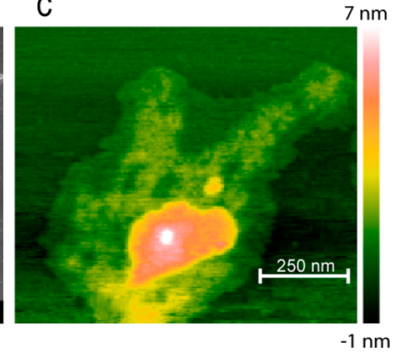

Figure 1. Dimensional and morphological analysis of NG: dimensional distribution obtained after 3 cycles of homogenization by dynamic light scattering (a), SEM micrograph of the dried sample (b), and AFM analysis of the swollen sample (c).

Table 1. Size (Z-Average), Polydispersity Index (PDI), ZPotential, and Percentage Swelling Degree (SD) of the Microgel and the Composite Nanogel (Mean \pm Std Dev, $n=$ 3)

\begin{tabular}{lcccc}
\multicolumn{1}{c}{ sample } & $Z$-average $(\mathrm{nm})$ & PDI & $Z$-potential $(\mathrm{mV})$ & $\mathrm{SD}(\%)$ \\
microgel & 1900 & 0.75 & $-17.1 \pm 1.4$ & 1680 \\
nanogel (NG) & 309 & 0.22 & $-23.8 \pm 0.8$ & 1770 \\
\hline
\end{tabular}

lateral size of about $250 \mathrm{~nm}$ inside the NG network (Figure 1c). AFM phase imaging is a powerful tool that is sensitive to surface stiffness and adhesion between the tip and surface. It allows mapping of surfaces based on these material differences. Thereby, phase imaging was employed to show that the local elastic modulus at the NG surface was quite heterogeneous, owing to the composite nature of the nanoparticles, varying approximately from 0.5 to $10 \mathrm{MPa}$. Overall, these data drop a hint that the proposed straightforward synthetic approach seems a reliable model, feasible at large production scales, to produce composite polygonal nanogels suitable for drug delivery applications.

Drug Loading and Physicochemical Characterization of the Nanogels. IT was chosen as a drug model for the treatment of colon carcinoma. It was loaded into the plain NG by simply placing NG in an aqueous solution of the free drug under sonication. Owing to the enormous swelling degree observed for NG (1770\%), which reached the maximum after about $2.5 \mathrm{~h}$ (Figure $2 \mathrm{a}$ ), the drug easily moved through the tortuous hydrogel network to be efficiently adsorbed on the GO surface. Indeed, the payload measured by HPLC analysis was $33 \%$ on a weight basis, corresponding to a loading efficiency of $100 \%$. The ability of the IT-loaded NG (IT/NG) of releasing its payload was assessed in PBS pH 7.4 and PBS $\mathrm{pH}$ 6.4 using the equilibrium dialysis method, mimicking physiological fluids and the tumor microenvironment, respectively (Figure 2b). The dialysis equilibrium time, previously determined employing an equivalent amount of IT hydrochloride at both $\mathrm{pH}$ values, was not dependent on the 
a)

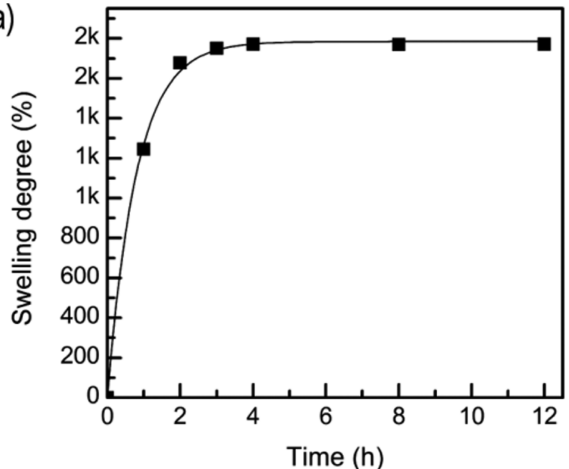

b)
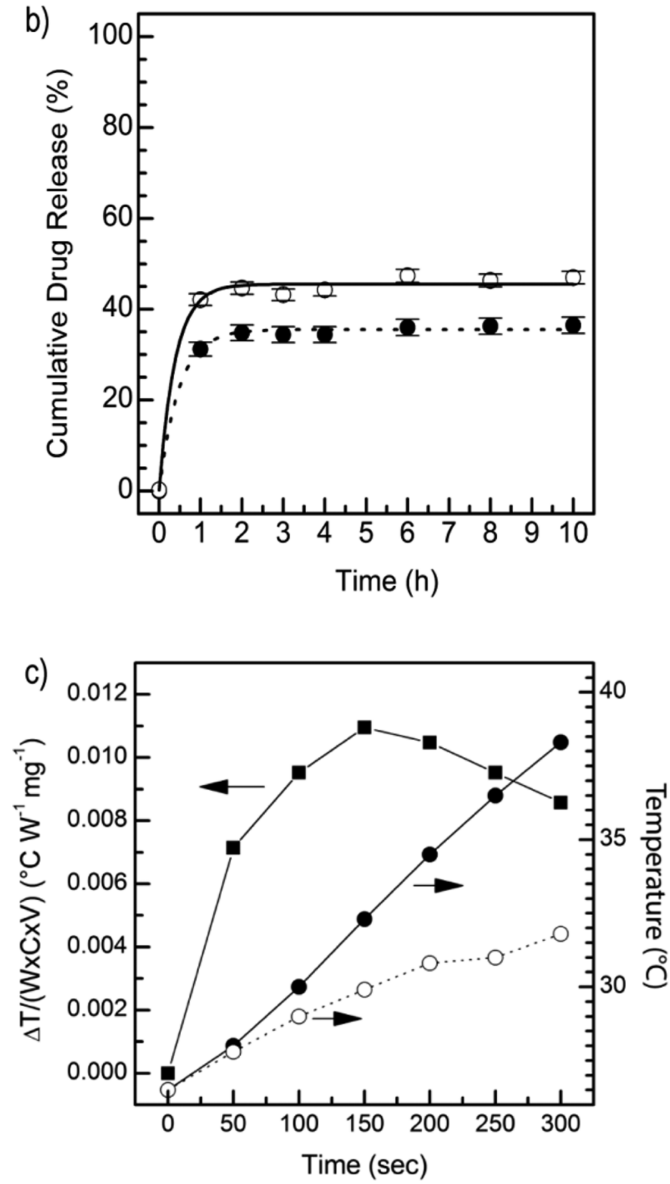

Figure 2. Swelling kinetics of NG in water (a). Drug release curves in PBS pH 6.4 (open symbol) and PBS 7.4 (solid symbol): the kinetic behavior is an exponential function model (b). Hyperthermal behavior of NG in water at concentration of $0.25 \mathrm{mg} \mathrm{mL}^{-1}$ (c): NG plot (solid circle), ultrapure water (open circle), and hyperthermia efficiency expressed as the increment of temperature normalized by the laser power, NG concentration and the sample volume (solid square).

medium and was reached after $1 \mathrm{~h}$. It might be noticed that IT/ NG released IT without a burst effect until an incubation time of $10 \mathrm{~h}$ at both $\mathrm{pH}$ considered, with a significant higher release at acidic conditions ( $42 \%$ vs $38 \%$ ). This is a good evidence that, under acidic conditions, the equilibrium of protonation of both the secondary amines $\left(\mathrm{p} K_{\mathrm{a}}\right)$ of the HA-EDA moieties and the carboxylic groups $\left(\mathrm{p} K_{\mathrm{a}}\right)$ of the GO nanosheets affects the arrangement of the polymer network. In particular, the reduction of the anionic character of the carboxylic groups, together with the increase in the number of cationic charges owing to the protonation of hindered amines, bring about reduction of electrostatic interaction throughout the hydrogel network, provoking a more efficient drug release in vitro. Moreover, having used GO flakes as carbon-based filler to introduce aromatic moieties in the polymeric network, it was not created a hydrophobic barrier inside the NG, maintaining a good percolation behavior in aqueous medium. The hyperthermia-mediated IT release was established irradiating a dispersion of IT/NG with a laser diode for 200 s. Noteworthy, $100 \%$ of the drug payload was released after $4 \mathrm{~h}$ incubation, pointing out that the local treatment with a cold laser may simultaneously trigger drug release and thermal ablation of the tumor mass.

The in vitro hyperthermal behavior of the NG suspension was assessed by following the kinetics of thermic gradient upon laser exposure for $300 \mathrm{~s}$ (Figure 2c). The temperature of the NG dispersion regularly increased with cumulative laser energy and, as expected, the heating rate was affected by the concentration of NG. In Figure $2 \mathrm{c}$ is also reported the trend of the hyperthermia efficiency (expressed as the $\mathrm{d} T / \mathrm{d} t$ normalized by laser power, NG concentration, and the sample volume) at each exposure time. As can be seen the hyperthermia efficiency increased with a remarkable timedependent trend until $150 \mathrm{~s}$, then slightly decreased. This evolution suggests a saturation of the vibration modes of GO flakes after $150 \mathrm{~s}$, which indicates that after this period the thermal dissipation overcomes vibrational effects bringing about a steady-state. As compared with other nano-GO materials with NIR light adsorption, NG exhibits a laser-triggered hyperthermia at least two times higher. The mass extinction coefficient for NG at $810 \mathrm{~nm}$ is $48.2 \mathrm{~L} \mathrm{~g}^{-1} \mathrm{~cm}^{-1}$, comparable to that of single-walled carbon nanotubes $\left(46.5 \mathrm{~L} \mathrm{~g}^{-1} \mathrm{~cm}^{-1}\right),{ }^{35}$ but roughly four times bigger than gold nanorods and two times than nano-RGO $\left(13.89\right.$ and $24.6 \mathrm{~L} \mathrm{~g}^{-1} \mathrm{~cm}^{-1}$, respectively). ${ }^{36}$

In Vitro Evaluation of the Cytotoxic Effect. The biological characterization of the produced NG alone or in the presence of IT (IT/NG) was conducted on human colorectal cancer cells HCT 116 since this is a growth factorindependent cell line invasive and highly motile in vitro. ${ }^{37}$

Cytotoxicity tests were conducted on HCT 116 incubating either NG or IT/NG suspension in DMEM at different concentrations. Figure $3 \mathrm{a}$ shows that NG alone was cytocompatible within the range of concentration tested, both after 4 and $24 \mathrm{~h}$ of incubation, since there is never a significant $(p<0.05)$ decrease of the cell viability compared to the untreated cells. These results clearly indicate that NG suspensions do not impair cells at high concentration $(1 \mathrm{mg}$ $\mathrm{mL}^{-1}$ ) neither. On the contrary, the presence of IT into the NG dramatically changed cell response toward the nanogel. In particular, for the drug-loaded NG (IT/NG) a remarked dosedependent cytotoxic effect was observed after $24 \mathrm{~h}$ of incubation, with an $\mathrm{EC}_{50}$ value almost two times lower than that observed for the free IT ( 150 vs $250 \mu \mathrm{g} \mathrm{mL}^{-1}$ respectively; Figure 3e). Notwithstanding this, compared with the control, after $4 \mathrm{~h}$ of incubation no significant $(p<0.05)$ viability decrease is observed for all the concentrations employed (see Figure S1 in the Supporting Information), suggesting that the higher potency displayed by IT/NG after $24 \mathrm{~h}$ might be correlated with biotransformation phenomena of IT occurring after cell internalization.

Evaluation of the In Vitro Hyperthermia of the Nanogels. The influence of the hypertermic effect on the HCT 116 viability was investigated by irradiating, for 100 and 


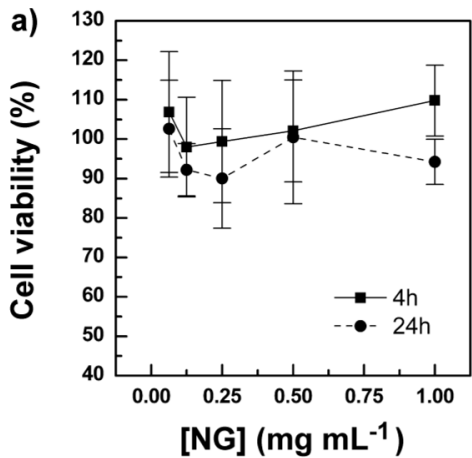

d)

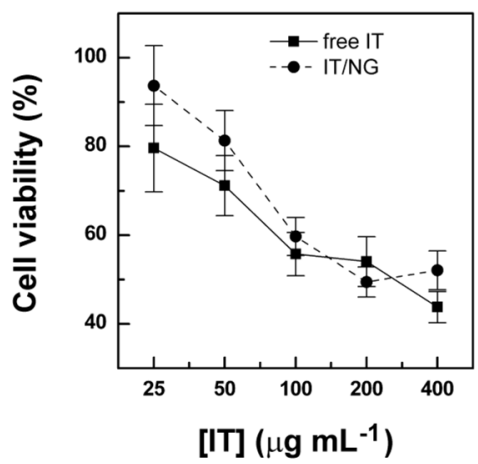

g)

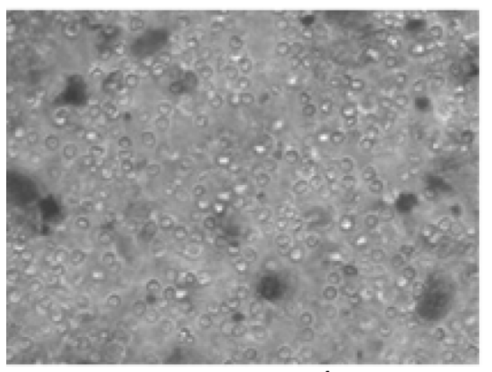

NG $1 \mathrm{mg} \mathrm{mL}^{-1}$

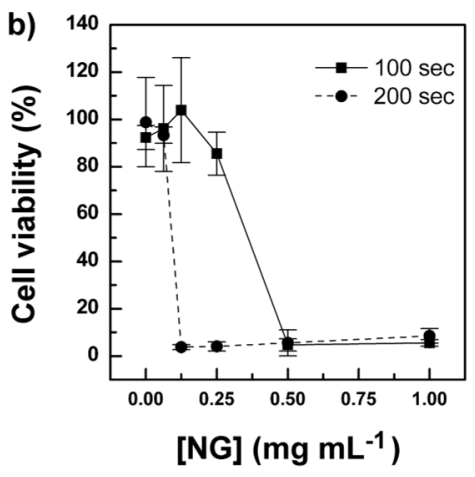

e)
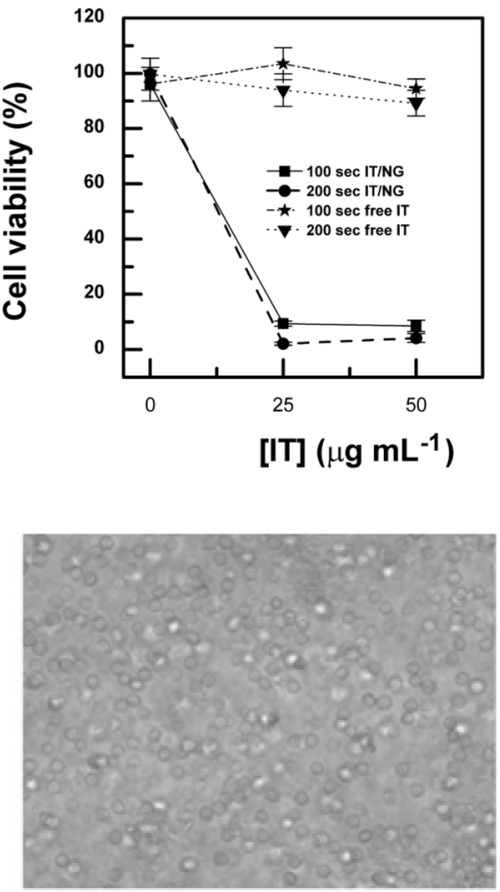

Negative Control

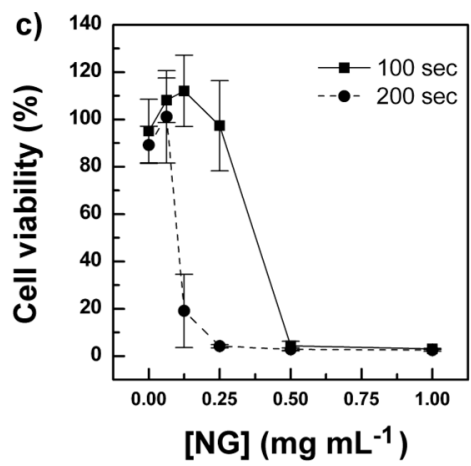

f)

\begin{tabular}{|lcc|}
\hline Sample & $\begin{array}{c}\mathrm{EC50} \\
\left(\mu \mathrm{g} \mathrm{mL}^{-1}\right)\end{array}$ & $\begin{array}{c}\mathrm{EC50} \\
\left(\mu \mathrm{g} \mathrm{mL}^{-1}\right)\end{array}$ \\
\hline NG & n.a. & 125 \\
\hline IT & 250 & n.a. \\
IT/NG & 150 & 12.5 \\
\hline
\end{tabular}

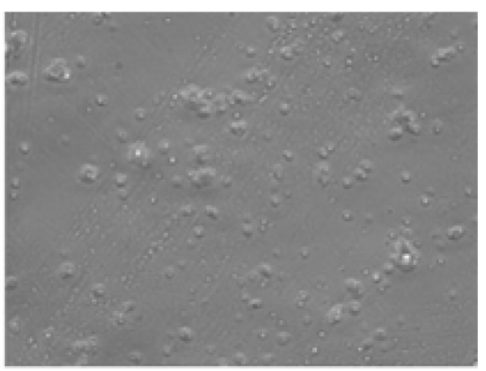

Positive Control

Figure 3. HCT 116 viability after 4 and $24 \mathrm{~h}$ of incubation with NG at different concentrations (a). Cell viability of HCT 116 irradiated for 100 and $200 \mathrm{~s}$ after $4 \mathrm{~h} \mathrm{(b)}$ and $24 \mathrm{~h}$ (c) of incubation with NG. HCT 116 viability after $24 \mathrm{~h}$ of incubation with free IT or IT/NG as a function of the IT concentration (d); HCT 116 viability treated with free IT and IT/NG for $4 \mathrm{~h}$ and irradiated for 100 and $200 \mathrm{~s}$ (e), EC s0 $_{50}$ values of the MTS experiments (f): *values calculated after laser exposure for $200 \mathrm{~s}$. Optical microscope images of erythrocytes treated with NG, untreated (negative control) and treated with Triton X 100 (positive control) (g).

$200 \mathrm{~s}$, cells cultured in the presence of NG suspensions at different concentrations (ranging from 0.0625 to $1 \mathrm{mg} \mathrm{mL}^{-1}$ ) for 4 and $24 \mathrm{~h}$. The irradiation time intervals have been chosen because, as already discussed, after these time there is the highest transformation efficiency of irradiated light into heat. No significant differences $(p<0.05)$ were noticed for the results obtained after the different scheduled times of incubation (Figure 3b,c). Suspensions with higher NG concentrations ( 0.5 and $1 \mathrm{mg} \mathrm{mL}^{-1}$ ) allowed almost a complete reduction in cell viability when irradiated both for 100 and $200 \mathrm{~s}$. The same effect is observed for 0.125 and 0.25 $\mathrm{mg} \mathrm{mL} \mathrm{m}^{-1} \mathrm{NG}$ suspensions but only when irradiated for $200 \mathrm{~s}$. In all the other cases no significant $(p<0.05)$ difference was observed in the cell viability compared to the control. These results show that HCT 116 are susceptible to the NG-mediated hyperthermic effect.

To evaluate if, as described in literature, ${ }^{20}$ the hyperthermic effect actually plays an adjuvant effect on IT toxicity, we conducted the hyperthermia experiment by irradiating HCT 116 cultured in the presence of IT/NG suspensions at the lower concentrations of IT employed for the cytotoxicity studies ( 25 and $50 \mu \mathrm{g} \mathrm{mL}^{-1}$ ). It is interesting to notice that, while IT/NG did not exhibit cytotoxic effects (Figures 3d), when subjected to the laser treatment it brings about an almost complete reduction of the cell viability already after $4 \mathrm{~h}$ of incubation (Figure 3e). This is a first confirmation that the hypertermic effect enhances the toxicity of IT incorporated into NG, demonstrated also by the fact that by irradiating free IT solutions it is not possible to cause any significant decrease in cell viability (Figure 3e). This leads us to state that there is a cumulative effect on the efficiency of the system mediated by hyperthermia, that is thermal ablation and enhanced IT activity. This behavior might be explained with the hyperthermiatriggered in situ formation of the metabolite 7-ethyl-10hydroxycamptothecin (SN-38), obtained upon cleaving the piperidino side-chain at the $\mathrm{C}-10$ position of IT by 
carboxylesterase, whose higher activity (1000-fold more potent than irinotecan $)^{38}$ implies increased average potency of the nanosystem after the laser exposure. It is worthy of note that IT/NG has an $\mathrm{EC}_{50}$ three times higher than the most effective data available in the literature panorama. For instance, ITloaded liposomes developed by Bo Zhang et al. ${ }^{39}$ display a $\mathrm{EC}_{50}$ of $60 \mu \mathrm{M}$. However, the $\mathrm{EC}_{50}$ of IT/NG reached a value 3 -fold lesser after the laser treatment $\left(12.5 \mu \mathrm{g} \mathrm{mL}^{-1} ; 20 \mu \mathrm{M}\right.$ ) (Figure 3f) endorsing the great therapeutic value of phototherapy using IT/NG. In addition, it should be highlighted that the drug loading of such liposomes rarely overtakes $2.5 \%$, implying that the amount of nanosystem employed to reach the therapeutic window must be 13-fold higher, with obvious drawbacks.

In Vitro Hemolysis Assay. The hemocompatibility of nanogels was tested on fresh erythrocytes suspension using a dose of NG ten times higher than that necessary for in vivo administration $\left(1 \mathrm{mg} \mathrm{mL} \mathrm{mL}^{-1}\right)$. As it possible to notice from Figure $3 \mathrm{~g}$, NG does not cause any damages to erythrocytes even at high concentration. This result is particularly important since, as we already discussed, the top-down production technique allows to obtain NG with dimension compatible with a possible intravenous administration and then, it is of crucial importance to demonstrate that our system does not promote any adverse effect on the cellular components of the blood.

In Vitro Photothermal Ablation on 3D Coculture Model. As a proof of concept, a 3D tumor model was assessed in vitro to demonstrate that NG could be eventually employed for the thermoablation of solid tumor masses. In this model we aimed to recreate the environment in which the cancer cells develop a tumor mass surrounded by healthy cells (see Figure S2 in the Supporting Information). The tumor microenvironment consists of key components contributing to tumor growth and proliferation such as various immune and inflammatory cells, blood, and lymphatic endothelial cells, cancer associated fibroblasts, and bone marrow-derived mesenchymal stem cells. $^{40,41}$ Among these cells, fibroblasts are particularly important in supporting the tumor growth since they promote angiogenesis, tumorigenicity, and metastatic dissemination of cancer cells. $^{42}$ In the tumor microenvironment cancer associated fibroblasts represent the major cell population and determine a poor intratumoral uptake of chemotherapeutic agents by the expression and organization of collagen type $\mathrm{I}^{43}$ NG or IT/NG suspensions were injected directly into the constructs with a $12.7 \mathrm{~mm}$ needle insulin syringe, in order to mimic a peri/intra tumor injection, and the samples were irradiated for $200 \mathrm{~s}$. Figure 4 shows that, in the NG treated construct (a), HCT 116 (green labeled cells) are well spread into the sample and do not show any sign of damage, while, in the IT/NG treated construct (b) both fibroblasts and HCT 116 seem to undergone a strong cytotoxic effect as it was possible to predict, even if not an exhaustive cell death is observed.

Interestingly, both $\mathrm{NG}$ and IT/NG treated constructs irradiated for $200 \mathrm{~s}$ with a $810 \mathrm{~nm}$ laser beam show a necrotic area in correspondence with the irradiation spot (Figure $4 c, d$ ) and this area is bigger for the construct treated with the IT/NG suspension (Figure 4d). Still, after $24 \mathrm{~h}$ of incubation, in the NG-treated construct, it is still possible to notice the presence of both cancer and healthy cells, while, a complete destruction of both cell type occurred when IT was present. These results again highlight the adjuvant effect of hyperthermia and IT cytotoxicity also in the thermoablation therapy.
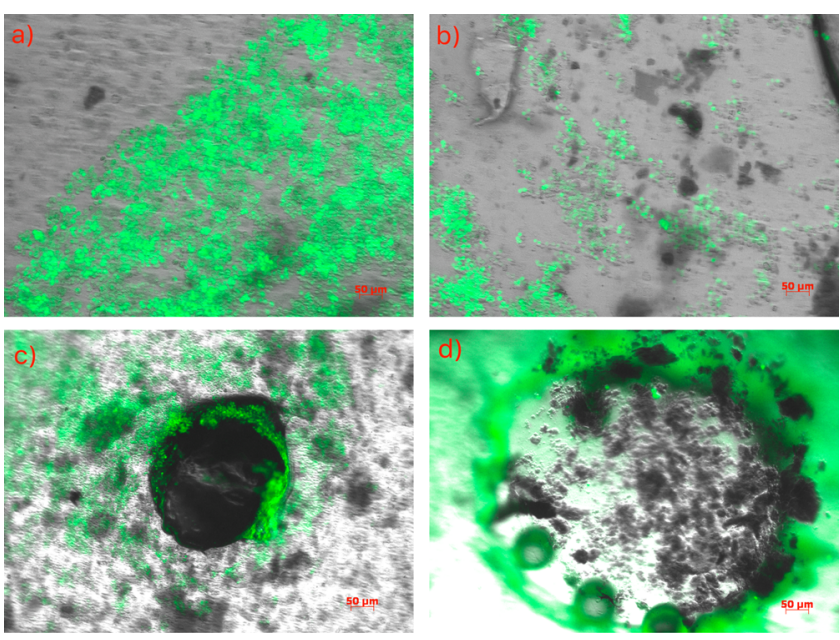

Figure 4. Brightfield and fluorescence merged images of HCT 116 (green) and HDF 3D matrigel constructs after $24 \mathrm{~h}$ of treatment with NG (a, c) or IT/NG (b, d) DMEM suspensions (NG concentration of $0.125 \mathrm{mg} \mathrm{mL}^{-1}$ ) nonirradiated $(\mathrm{a}, \mathrm{b})$ or irradiated for $200 \mathrm{~s}$ with 810 $\mathrm{nm}$ laser beam (c, d).

Cellular Uptake of Cy5-Labeled NG on HCT 116 Cells. As above-discussed, the metabolite $\mathrm{SN}-38$ obtained upon cleaving the piperidino side-chain at the $\mathrm{C}-10$ position of IT is 1000 -fold more potent than irinotecan. Thus, SN-38 generated inside cells or near the cell membrane might display greater activity as compared to $\mathrm{SN}-38$ generated outside the cells in the culture medium. ${ }^{44}$ To demonstrate that NG could be internalized by cancer cells so as to allow the production of SN-38 inside cells, with obvious implications, uptake studies were conducted by incubating the HCT 116 with of Cy-5 labeled NG suspension. Figure 5 shows that NG enter cells already after $4 \mathrm{~h}$ of incubation and appear uniformly distributed throughout the cytoplasm after $24 \mathrm{~h}$. This behavior explains well the higher cytotoxicity observed after $24 \mathrm{~h}$ incubation with IT/NG, especially after the photothermal treatment.

The uptake of the CY5-labeled nanosystem was also studied by flow cytometry analysis on a culture of HTC 116. Figure 6a shows the medium fluorescence intensity (MFI) of cells after the incubation with NG for 4 and $24 \mathrm{~h}$. It can be seen a right shift of the entire cell population after $4 \mathrm{~h}$ incubation, with a remarked difference observed after $24 \mathrm{~h}$, implying that NG homogeneously enter inside cells in a time-dependent fashion. The relation between uptake and incubation time is more clear in Figure 6b, where a significant increase in cell fluorescence is observed over time (from 1.5 to 4.0, $p<0.05$, for the control and after $24 \mathrm{~h}$ incubation, respectively). On the whole, these data corroborate the fluorescence microscopy analysis, highlighting that NG is able to enter cells thereby releasing its drug payload inside cancer cells.

\section{CONCLUSIONS}

In this paper, the Michael-type addition of an aminofunctionalized hyaluronic acid with an acryloyl-functionalized polyamino acid in the presence of single layer graphene oxide (GO) flakes to give a double-network-structured nanogel, henceforth NG, is reported. This nanogel represents a relevant example of biomimetic hybrid material capable of incorporating and releasing in a controlled manner poorly water-soluble antitumor drugs such as irinotecan (IT). The top-down method adopted to obtain NG allowed reaching fairly nanogels with 

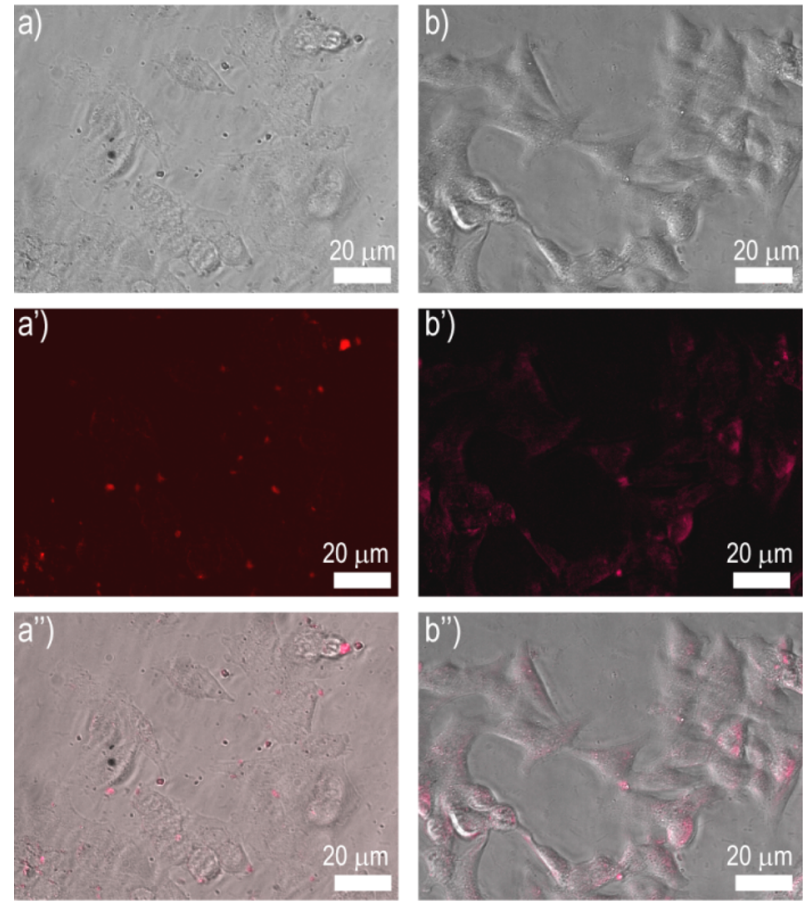

Figure 5. Uptake of NG on HCT 116 after 4 h (a, a $\left.a^{\prime} a^{\prime \prime}\right)$ and 24 h (b, $\left.b^{\prime}, b^{\prime \prime}\right)$ of incubation with Cy5-labeled NG suspensions at concentration of $0.25 \mathrm{mg} \mathrm{mL}^{-1}$ : brightfield $(a, b)$, fluorescence $\left(a^{\prime}\right.$, $\left.\mathrm{b}^{\prime}\right)$, and merged $\left(\mathrm{a}^{\prime \prime}, \mathrm{b}^{\prime \prime}\right)$.

a)

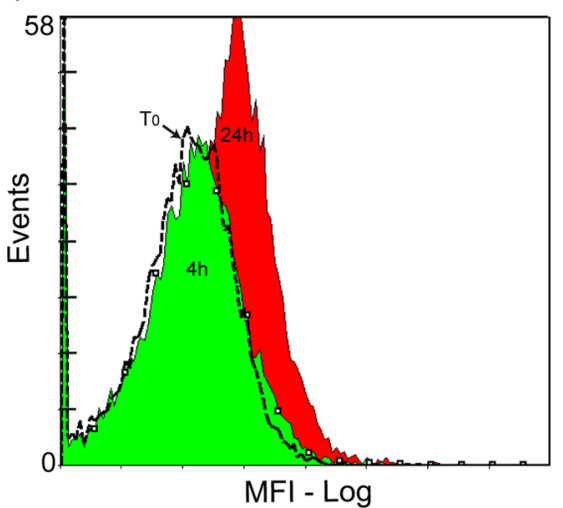

b)

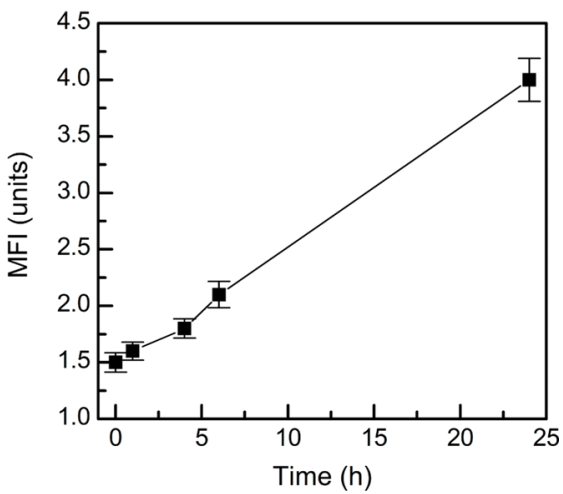

Figure 6. Relationship between uptake and incubation time on HCT 116 by flow cytometry analysis. Population of fluorescent cells as a function of medium fluorescence intensity per cell (a); medium fluorescence intensity (MFI) against incubation time (b). good size distribution and outstanding near-infrared (NIR) light-induced hyperthermia useful in photothermal therapy. Here, we demonstrated that IT-loaded NG, owing to the presence of hyaluronic acid and GO sheets, is endowed with an adjuvant effect between hyperthermia and chemotherapy, displaying in the meantime remarkable biocompatibility. Finally, we showed that IT-loaded NG can be employed for the photothermal ablation of solid tumors after local injection, pointing out that this hybrid nanogel can be employed as dual action mode anticancer tool.

\section{ASSOCIATED CONTENT}

\section{Supporting Information}

The Supporting Information is available free of charge on the ACS Publications website at DOI: 10.1021/acs.biomac.6b01897.

HCT 116 viability after $4 \mathrm{~h}$ of incubation with free IT and IT/NG, HCT 116, and HDF 3D Matrigel coculture (PDF).

\section{AUTHOR INFORMATION}

\section{Corresponding Author}

*E-mail: nicolo.mauro@unipa.it. Fax: +39 09123891928. Tel.: +3909123891928.

\section{ORCID}

Nicolò Mauro: 0000-0003-0246-3474

\section{Author Contributions}

C.F. performed the biological experiments, analyzed the results, and helped to write the manuscript. N.M. conceived and managed the project, synthesized the hydrogel, performed and analyzed all chemical and physicochemical characterizations, and wrote the main manuscript. G.P. contributed to write the manuscript. C.S. contributed to perform biological experiments. F.S.P. revised all figures and tables. G.G. contributed to manage the project and revised the final manuscript. All authors reviewed and approved the final manuscript.

Notes

The authors declare no competing financial interest.

\section{REFERENCES}

(1) Ferlay, J.; Soerjomataram, I.; Dikshit, R.; Eser, S.; Mathers, C.; Rebelo, M.; Parkin, D. M.; Forman, D.; Bray, F. Int. J. Cancer 2015, 136 (5), E359-E386.

(2) Mengual-Ballester, M.; Pellicer-Franco, E.; Valero-Navarro, G.; Soria-Aledo, V.; Andrés García-Marín, J.; Aguayo-Albasini, J. L. Cancer Epidemiol. 2016, 43, 70-75.

(3) Oh, H.-K.; Ihn, M. H.; Son, I. T.; Park, J. T.; Lee, J.; Kim, D.-W.; Kang, S.-B. Surg. Endosc. 2016, 30 (3), 1086-1093.

(4) Anitha, A.; Maya, S.; Sivaram, A. J.; Mony, U.; Jayakumar, R. Wiley Interdiscip. Rev. Nanomed. Nanobiotechnol. 2016, 8 (1), 151-159.

(5) Viswanath, B.; Kim, S.; Lee, K. Int. J. Nanomed. 2016, 11, 24912504.

(6) Juliano, R. Nat. Rev. Drug Discovery 2013, 12 (3), 171-172.

(7) Mura, S.; Nicolas, J.; Couvreur, P. Nat. Mater. 2013, 12 (11), 991-1003.

(8) Mauro, N.; Campora, S.; Adamo, G.; Scialabba, C.; Ghersi, G.; Giammona, G. RSC Adv. 2016, 6 (81), 77256-77266.

(9) Mauro, N.; Campora, S.; Scialabba, C.; Adamo, G.; Licciardi, M.; Ghersi, G.; Giammona, G. RSC Adv. 2015, 5 (41), 32421-32430.

(10) Muzzalupo, R.; Tavano, L.; Cassano, R.; Trombino, S.; Cilea, A.; Picci, N. Eur. Polym. J. 2010, 46 (2), 209-216.

(11) Laurent, T. C. Acta Oto-Laryngol. 1987, 104, 7-24. 
(12) Knudson, C. B.; Knudson, W. FASEB J. 1993, 7 (13), 12331241

(13) Entwistle, J.; Hall, C. L.; Turley, E. A. J. Cell. Biochem. 1996, 61 (4), 569-577.

(14) Peterson, R. M.; Yu, Q.; Stamenkovic, I.; Toole, B. P. Am. J. Pathol. 2000, 156 (6), 2159-2167.

(15) Carbary-Ganz, J. L.; Welge, W. A.; Barton, J. K.; Utzinger, U. J. Biomed. Opt. 2015, 20 (9), 96015.

(16) Mauro, N.; Li Volsi, A.; Scialabba, C.; Licciardi, M.; Cavallaro, G.; Giammona, G. Curr. Drug Delivery 2016, 13 (8), 1.

(17) Yang, D.; Feng, L.; Dougherty, C. A.; Luker, K. E.; Chen, D.; Cauble, M. A.; Banaszak Holl, M. M.; Luker, G. D.; Ross, B. D.; Liu, Z.; Hong, H. Biomaterials 2016, 104, 361-371.

(18) Vila, M.; Matesanz, M. C.; Gonçalves, G.; Feito, M. J.; Linares, J.; Marques, P. A. A. P.; Portolés, M. T.; Vallet-Regi, M. Nanotechnology 2014, 25 (3), 35101.

(19) Mauro, N.; Scialabba, C.; Cavallaro, G.; Licciardi, M.; Giammona, G. Biomacromolecules 2015, 16 (9), 2766-2775.

(20) Cotte, E.; Passot, G.; Tod, M.; Bakrin, N.; Gilly, F.-N.; Steghens, A.; Mohamed, F.; Glehen, O. Ann. Surg. Oncol. 2011, 18 (9), 25992603.

(21) Gottesman, M. M. Annu. Rev. Med. 2002, 53 (1), 615-627.

(22) Palumbo, F. S.; Pitarresi, G.; Fiorica, C.; Matricardi, P.; Albanese, A.; Giammona, G. Soft Matter 2012, 8 (18), 4918.

(23) Fiorica, C.; Palumbo, F. S.; Pitarresi, G.; Gulino, A.; Agnello, S.; Giammona, G. RSC Adv. 2015, 5 (25), 19715-19723.

(24) Allegra, M.; D’acquisto, F.; Tesoriere, L.; Attanzio, A.; Livrea, M. A. Redox Biol. 2014, 2 (2), 892-890.

(25) Peppas, N. A.; Bures, P.; Leobandung, W.; Ichikawa, H. Eur. J. Pharm. Biopharm. 2000, 50 (1), 27-46.

(26) Raemdonck, K.; Demeester, J.; De Smedt, S. Soft Matter 2009, 5 (4), 707-715.

(27) Toita, S.; Sawada, S.; Akiyoshi, K. J. Controlled Release 2011, 155 (1), 54-59.

(28) Liechty, W. B.; Peppas, N. A. Eur. J. Pharm. Biopharm. 2012, 80 (2), 241-246.

(29) Soni, G.; Yadav, K. S. Pharm. Dev. Technol. 2014, 19 (6), 651661.

(30) Oh, J. K.; Siegwart, D. J.; Lee, H.; Sherwood, G.; Peteanu, L.; Hollinger, J. O.; Kataoka, K.; Matyjaszewski, K. J. Am. Chem. Soc. 2007, 129 (18), 5939-5945.

(31) Misra, S.; Heldin, P.; Hascall, V. C.; Karamanos, N. K.; Skandalis, S. S.; Markwald, R. R.; Ghatak, S. FEBS J. 2011, 278 (9), 1429-1443.

(32) Peiris, P. M.; Bauer, L.; Toy, R.; Tran, E.; Pansky, J.; Doolittle, E.; Schmidt, E.; Hayden, E.; Mayer, A.; Keri, R. A.; Griswold, M. A.; Karathanasis, E. ACS Nano 2012, 6 (5), 4157-4168.

(33) Toy, R.; Peiris, P. M.; Ghaghada, K. B.; Karathanasis, E. Nanomedicine (London, U. K.) 2014, 9 (1), 121-134.

(34) Gabizon, A.; Papahadjopoulos, D. Proc. Natl. Acad. Sci. U. S. A. 1988, 85 (18), 6949-6953.

(35) Robinson, J. T.; Welsher, K.; Tabakman, S. M.; Sherlock, S. P.; Wang, H.; Luong, R; Dai, H. Nano Res. 2010, 3 (11), 779-793.

(36) Robinson, J. T.; Tabakman, S. M.; Liang, Y.; Wang, H.; Sanchez Casalongue, H.; Vinh, D.; Dai, H. J. Am. Chem. Soc. 2011, 133 (17), 6825-6831.

(37) Rajput, A.; Dominguez San Martin, I.; Rose, R.; Beko, A.; LeVea, C.; Sharratt, E.; Mazurchuk, R.; Hoffman, R. M.; Brattain, M. G.; Wang, J. J. Surg. Res. 2008, 147, 276-281.

(38) Kunimoto, T.; Nitta, K.; Tanaka, T.; Uehara, N.; Baba, H.; Takeuchi, M.; Yokokura, T.; Sawada, S.; Miyasaka, T.; Mutai, M. Cancer Res. 1987, 47 (22), 5944-5947.

(39) Zhang, B.; Wang, T.; Yang, S.; Xiao, Y.; Song, Y.; Zhang, N.; Garg, S. J. Controlled Release 2016, 238, 10-21.

(40) Kemp, J. A.; Shim, M. S.; Heo, C. Y. Adv. Drug Delivery Rev. 2016, 98, 3-18.

(41) Sounni, N. E.; Noel, A. Clin. Chem. 2013, 59 (1), 85-93.

(42) Erez, N.; Truitt, M.; Olson, P.; Hanahan, D. Cancer Cell 2010, 17 (2), 135-147.
(43) Baronzio, G.; Parmar, G.; Baronzio, M. Front. Oncol. 2015, 5, 115 .

(44) Hsieh, Y.-T.; Lin, H.-P.; Chen, B.-M.; Huang, P.-T.; Roffler, S. R. PLoS One 2015, 10 (10), e0141088. 